\title{
A Sporadic Neonatal Case of Epidermolysis Bullosa Simplex Generalized Intermediate with KRT5 and KRT14 Gene Mutations
}

Hiroyuki Wakiguchi, MD, PhD ${ }^{1}$ Shunji Hasegawa, MD, $\mathrm{PhD}^{1}$ Shinji Maeba, MD, $\mathrm{PhD}^{1}$ Sasagu Kimura, MD ${ }^{1}$ Satoko Ito, MD $^{2}$ Hiroshi Tateishi, MD, $\mathrm{PhD}^{2}$ Kazuhiro Ueda, MD, $\mathrm{PhD}^{3}$ Shouichi Ohga, MD, $\mathrm{PhD}^{1}$

1 Department of Pediatrics, Graduate School of Medicine, Yamaguchi
University, Ube, Yamaguchi, Japan
${ }^{2}$ Division of Pediatrics, JCHO Tokuyama Central Hospital, Shunan,
Yamaguchi, Japan
${ }^{3}$ Division of Pediatrics, Michigami Hospital, Hikari, Yamaguchi, Japan Am J Perinatol Rep 2016;6:e108-e111.
Address for correspondence Shunji Hasegawa, MD, PhD, Department of Pediatrics, Graduate School of Medicine, Yamaguchi University, 1-11 Minamikogushi, Ube, Yamaguchi 755-8505, Japan (e-mail: shunji@yamaguchi-u.ac.jp).

\begin{abstract}
Background Epidermolysis bullosa simplex (EBS) is a rare genodermatosis resulting from multiple gene mutations, including KRT5 and KRT14. The clinical expression of the mechanobullous skin fragility disease has not been fully explained by the genotype. Case Description An 11-day-old Japanese newborn infant was hospitalized because of herpetiform skin blistering on the feet, which expanded systemically after birth. There was no evidence of virus infection. The biopsied skin lesion showed a blister on the lamina densa without keratin clumps, indicating a diagnosis of EBS-generalized intermediate. We punctured the blisters to remove the contents daily, which led to no exacerbation or infection. The genetic study determined that the patient carried

Keywords

- epidermolysis bullosa simplex

- KRT5

- KRT14

- mutation

- newborn double substitutions of KRT5 c.1424A > G (p.E475G) and KRT14 c.1237G > A (p.A413T). The asymptomatic mother and sister carried the KRT14 substitution, but the healthy father had no substitution of the KRT gene.

Conclusion This is the first report of EBS-generalized intermediate in a newborn with de novo KRT5 gene mutation and KRT14 gene polymorphism, and no familial history of epidermolysis. Neonatal blistering due to EBS requires optimal skin management after excluding infectious and immunobullous diseases.
\end{abstract}

Epidermolysis bullosa simplex (EBS) is a rare genodermatosis resulting from TGM5, DSP, JUP, PKP1, KRT5, KRT14, EXPH5, PLEC, or DST gene mutations. The mechanobullous skin fragility disease is characterized by cytolysis of basal keratinocytes and intraepidermal blistering. ${ }^{1,2}$ EBS is classified into three major types of disease based on distinctive clinical features of KRT5 and KRT14 gene mutations. ${ }^{3,4}$ In patients with the EBS-localized variant (EBS-loc and Weber-Cockayne type), bullous lesions may present as late as the third decade of life, but they are confined to the hands and feet. In patients with the EBS-generalized intermediate variant (EBS-gen

received

July 12,2015 accepted after revision November 13, 2015
DOI http://dx.doi.org/ $10.1055 / \mathrm{s}-0035-1570386$. ISSN 2157-6998. intermed and Köebner type), widespread blistering, with occasional involvement of the oral mucosa and nails, begin from infancy. The EBS-generalized severe variant (EBS-gen sev and Dowling-Meara type) is the most severe form in newborns, presenting with generalized blistering of the skin, which is accompanied by oral mucosa bullae and nail shedding. ${ }^{3,4}$ Neonatal herpes simplex virus infection is a critical diagnosis to differentiate from systemic EBS occurring in newborns with no familial history of blistering.

We present a newborn infant with EBS-gen intermed who had systemic blistering after birth. He had no family history of
Copyright $\odot 2016$ by Thieme Medical Publishers, Inc., 333 Seventh Avenue, New York, NY 10001, USA. Tel: +1(212) 584-4662.
License terms

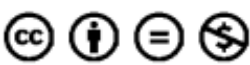


genodermatosis but carried the KRT5 and KRT14 gene substitutions. A family study determined that de novo KRT5, but not the KRT14 substitution, was the pathognomonic finding for the disease. The early genetic study and skin management successfully controlled the systemic blistering.

\section{Case Description}

An 11-day-old Japanese newborn infant was transferred to the neonatal intensive care unit because of systemic skin blisters. He was the second-born child of healthy, unrelated Japanese parents, and there was no familial history of blistering. He was normal and delivered vaginally, weighing 3,380 g at 40 weeks of gestation and was suspected of having EBS because of localized skin blistering on the feet at birth. There was no evidence of bacterial, viral, and fungal infections, including the herpes simplex virus and varicella virus, along with immunobullous diseases according to the diagnostic algorithm of blisters and erosions in newborns. ${ }^{5}$ One day after birth, the skin lesions extended to the infant's face, hands, and feet after mechanical stresses. Nikolsky sign was positive. He had blisters in the oral membranes, which tended to heal without scars. Eleven days after birth, the blisters became generalized, followed by blistering on the head, neck, upper and lower limbs, nipples, navel, and buttocks (-Fig. 1A, B).

On admission, a skin biopsy of the blisters was performed with electron microscopy. It showed a blister on the lamina densa without keratin clumps (-Fig. 1C). We excluded gastrointestinal (no bloody stool), respiratory (neither symptoms nor radiographic findings), and ocular (neither symptoms nor clinical findings) involvement. These findings led to the diagnosis of EBS-gen intermed. We treated him as follows. Smooth materials were chosen for his bed sheets to avoid mechanical stress. A nipple comprised a soft material for cleft palate was selected for his nursing bottle. Vaseline was applied to his skin to reduce friction. We punctured the blisters and removed the contents daily. Bullous lesions remained, but they did not become widespread. Because of no exacerbation, he was discharged from the hospital 72 days

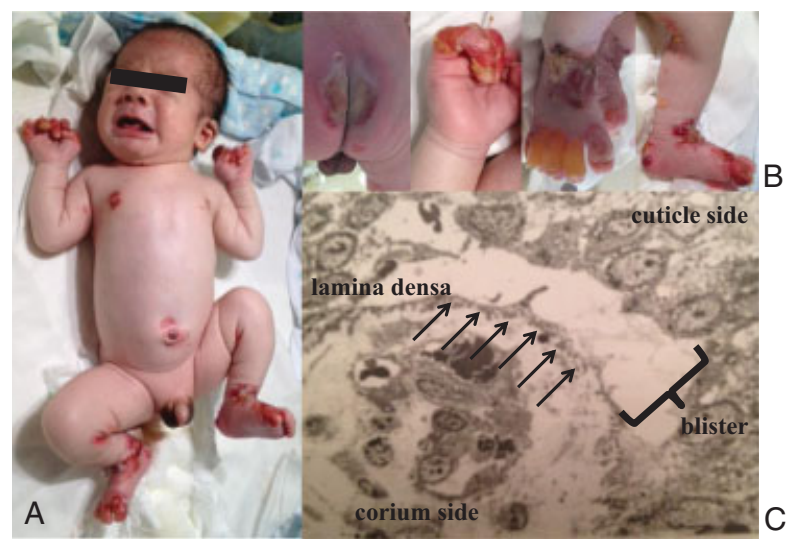

Fig. 1 Clinical presentation and electron microscopy of the patient with epidermolysis bullosa simplex. (A) Blisters at 11 days old. (B) Blistering of the buttocks, right hand, and feet. (C) Ultrastructural features of the proband's lesional skin sample show a blister on the lamina densa. There are no apparent keratin clumps. after birth. Subcutaneous injections of inactivated vaccines (Haemophilis influenzae type b, Pneumococcus, diphtheria, pertussis, tetanus, and polio) and live vaccines (measles, rubella, and varicella) precipitated no skin blistering. His generalized skin blisters were controlled without exacerbation and cutaneous union. He showed normal growth and development, including the development of teeth at 1 year old.

The genetic study of proband determined double substitutions of c.1424A > G (p.E475G) in the KRT5 gene and c.1237G > A (p.A413T) in the KRT14 gene (-Fig. 2). The infant's father carried neither the KRT5 gene mutation nor the KRT14 gene substitutions. However, his mother and sister had c.1237G $>\mathrm{A}$ in the KRT14 gene, but not the KRT5 gene mutation (-Fig. 3). His parents and elder sister showed no skin disease.

\section{Discussion}

We found an EBS-gen intermed newborn infant with KRT5 and KRT14 gene substitutions and unaffected family members. Systemic EBS should be distinguished from other ominous diseases of neonatal blistering, including the herpes simplex virus infection. The shapes, size, and distribution of blisters as well as a positive family history are important for the diagnosis of EBS. In the present case, the clinical expressions and findings of electron microscopy showed a blister on the lamina densa without keratin clumps, which led to the diagnosis of EBS-gen intermed. 6,7

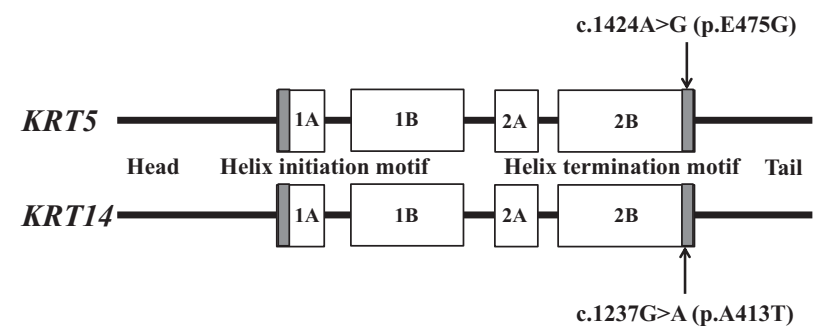

Fig. 2 Mutation sites of the patient with epidermolysis bullosa simplex. The $\mathrm{N}$ - and $\mathrm{C}$-terminus of the $\alpha$-helical rod domain (white boxes, 1A, 1B, 2A, and 2B) are the helix initiation and termination motifs, respectively (gray boxes). The arrows denote the positions of the point mutation in KRT5 and KRT14 in the patient.

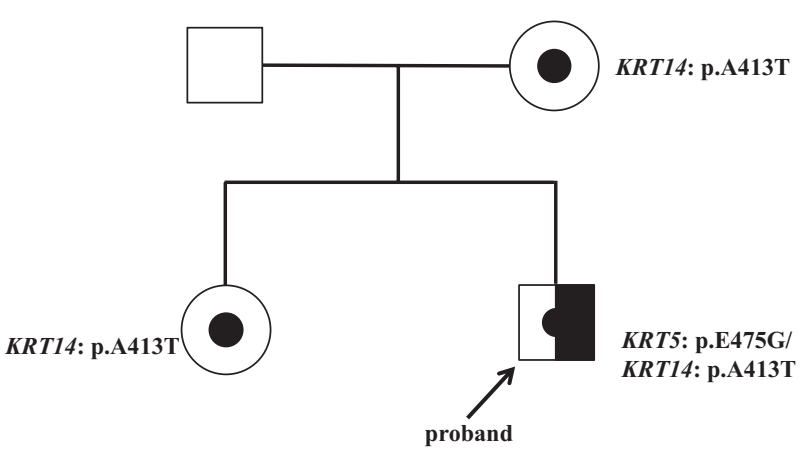

Fig. 3 Pedigree of the patient with epidermolysis bullosa simplex. 
Because of no cure for EBS, careful management is required to prevent the formation of new blisters and infections. ${ }^{8}$ We chose smooth materials for his bed sheets and a soft nipple for his nursing bottle, and applied petroleum jelly to his skin, which all helped reduce friction on his skin and oral mucosa. In our observation, puncturing the blisters daily to remove the contents, including old keratins, may have accelerated wound healing. Effective care during the newborn period prevents long-lasting cosmetic and functional complications.

The incidence of de novo mutation in patients with EBS reportedly ranges from 37 to $58 \%{ }^{9-11}$ In patients with EBS-gen intermed, de novo mutations may develop more frequently $(\sim 70 \%){ }^{9-11}$ Our patient had two substitutions of c.1424A > G (p.E475G) in the KRT5 gene and c.1237G > A (p.A413T) in the KRT14 gene. The asymptomatic mother and sister carried c.1237G > A in the KRT14 gene, but not the KRT5 gene mutation. Clinical investigation did not reveal minor trauma-induced blisters on their hands and feet. The KRT5 substitution has been reported in patients with EBS-gen sev, but not in the other types of EBS. ${ }^{12}$ Histopathology did not show circinate blisters and keratin clumps that are characteristic of EBS-gen sev. ${ }^{6,7}$ In addition, his blisters were not localized in the hands and feet as in the case of EBS-loc. ${ }^{3}$

This is the first report of EBS-gen intermed due to KRT5 c.1424A > G. The PolyPhen-2 program (http://genetics. bwh.harvard.edu/pph2/) predicted that c.1424A $>\mathrm{G}$ in KRT5 and the c.1237G $>$ A polymorphism were probably causing damage to protein function with a score of 0.854 and 0.964 , respectively (benign: $0.00-0.20$, possibly damaging: $0.20-0.85$, probably damaging: $0.85-1.00$ ). The c.1237G > A in KRT14 may contribute, if any, to the early expression of our patient as a modifier of EBS. The substitution c. $1237 \mathrm{G}>\mathrm{A}$ in KRT14 may be a polymorphism ${ }^{2}$ or an unknown pairwise transcriptional regulation of KRT5 and KRT14 genes may exist, although heterozygous mutation of KRT5 c. $1424 \mathrm{~A}>\mathrm{G}$ alone can dominantly cause clinical manifestations of EBS. ${ }^{1}$ Wertheim-Tysarowska et al ${ }^{13}$ reported that a 3-year-old patient with EBS-gen intermed had the coexistence of c.1412G > A (p.R471H) in KRT5 and c.815T > C (p.M272T) in KRT14 mutations. The proband's father and mother had c.815T > C in KRT14 and c.1412G $>\mathrm{A}$ in KRT5, respectively, and both were diagnosed as having EBS-loc. They concluded that c.1412G > A in KRT5 may act in a dominant-negative manner and the mutation leads to mild skin fragility when present alone, but in the presence of a mutation in the other keratin, it exacerbates the symptoms. On the contrary, the genotype-phenotype correlations of EBS have been reported to rely heavily on " the mutation location" in either KRT5 or KRT14. ${ }^{14}$ Mutations altering highly conserved residues located within the helix termination motifs in KRT5 are overwhelmingly associated with EBS-gen sev. Patients with EBS-gen intermed and EBS-loc are more frequently caused by KRT14 mutations than KRT5 mutations in the helix termination motifs. $^{14}$ The present patient carried KRT5 and KRT14 mutations in the helix termination motifs ( - Fig. 2). These results suggested that c.1424A > G in KRT5 led to EBS-gen sev when present alone, but in the presence of c. $1237 \mathrm{G}>\mathrm{A}$ in KRT14, it alleviated the symptoms.

\section{Conclusion}

We reported the first newborn infant with EBS-gen intermed carrying the de novo KRT5 gene mutation and KRT14 gene polymorphism. The neonatal diagnosis of EBS is critical if there is no useful information on the family history.

\section{Competing Interests}

The authors declare that they have no competing interests.

\section{Consent}

Informed consent was obtained from the patient's legal guardian for publication of this case report.

\section{Acknowledgments}

We thank Prof. Masahiko Muto, Dr. Michiya Yamaguchi, and Dr. Kishiko Matsumoto (Department of Dermatology, Yamaguchi University Graduate School of Medicine) for performing the genetic study.

\section{References}

1 Chamcheu JC, Virtanen M, Navsaria H, Bowden PE, Vahlquist A, Törmä H. Epidermolysis bullosa simplex due to KRT5 mutations: mutation-related differences in cellular fragility and the protective effects of trimethylamine $\mathrm{N}$-oxide in cultured primary keratinocytes. Br J Dermatol 2010;162(5):980-989

2 Natsuga K, Nishie W, Smith BJ, et al. Consequences of two different amino-acid substitutions at the same codon in KRT14 indicate definitive roles of structural distortion in epidermolysis bullosa simplex pathogenesis. J Invest Dermatol 2011;131(9):1869-1876

3 Premaratne C, Klingberg S, Glass I, Wright K, Murrell D. Epidermolysis bullosa simplex Dowling-Meara due to an arginine to cysteine substitution in exon 1 of keratin 14. Australas J Dermatol 2002;43(1):28-34

4 Fine JD, Bruckner-Tuderman L, Eady RA, et al. Inherited epidermolysis bullosa: updated recommendations on diagnosis and classification. J Am Acad Dermatol 2014;70(6):1103-1126

5 Nischler E, Klausegger A, Huttner C, et al. Diagnostic algorithm of blisters and erosions in newborns. Dermatol Res Pract 2009; 2009:320403

6 Anton-Lamprecht I, Schnyder UW. Epidermolysis bullosa herpetiformis Dowling-Meara. Report of a case and pathomorphogenesis. Dermatologica 1982;164(4):221-235

7 Fine JD, Eady RA, Bauer EA, et al. Revised classification system for inherited epidermolysis bullosa: Report of the Second International Consensus Meeting on diagnosis and classification of epidermolysis bullosa. J Am Acad Dermatol 2000;42(6):1051-1066

8 Gonzalez ME. Evaluation and treatment of the newborn with epidermolysis bullosa. Semin Perinatol 2013;37(1):32-39

9 Bolling MC, Lemmink HH, Jansen GH, Jonkman MF. Mutations in KRT5 and KRT14 cause epidermolysis bullosa simplex in 75\% of the patients. Br J Dermatol 2011;164(3):637-644

10 Kang TW, Lee JS, Kim SE, Oh SW, Kim SC. Novel and recurrent mutations in Keratin 5 and 14 in Korean patients with 
Epidermolysis bullosa simplex. J Dermatol Sci 2010;57(2): 90-94

11 Minakawa S, Nakano H, Nakajima K, et al. Mutational analysis on 16 Japanese population cases with epidermolysis bullosa simplex. J Dermatol Sci 2013;72(3):330-332

12 Lane EB, Rugg EL, Navsaria H, et al. A mutation in the conserved helix termination peptide of keratin 5 in hereditary skin blistering. Nature 1992;356(6366):244-246
13 Wertheim-Tysarowska K, Sota J, Kutkowska-Kaźmierczak A, Woźniak K, Bal J, Kowalewski C. Coexistence of KRT14 and KRT5 mutations in a Polish patient with epidermolysis bullosa simplex. Br J Dermatol 2014;170(2): 468-469

14 Coulombe PA, Kerns ML, Fuchs E. Epidermolysis bullosa simplex: a paradigm for disorders of tissue fragility. J Clin Invest 2009;119(7): 1784-1793 